\title{
FRAGILIDADE, COGNIÇÃO, DEPRESSÃO E FUNCIONALIDADE DE IDOSOS EM CONDOMINIO HABITACIONAL
}

\author{
Juliana Cerqueira Leite ${ }^{1}$ \\ Isabela Thaís Machado de Jesus² \\ Fabiana Souza Orlandi ${ }^{3}$ \\ Marisa Silvana Zazzetta ${ }^{4}$
}

resumo

Objetivo: Identificar a relação da fragilidade com cognição, depressão e capacidade funcional de residentes em condomínio exclusivo para idosos no interior do estado de São Paulo, Brasil. Método: Estudo transversal, com utilização do método quantitativo. Utilizaram-se da entrevista semiestruturada, do fenótipo de fragilidade proposto por Fried, do Mini Exame do Estado Mental, da Escala de Depressão Geriátrica, Índice de

1 Graduada em Gerontologia. Mestre em Ciências da Saúde. E-mail: juliana_cleite@yahoo.com.br.

2 Graduada em Gerontologia. Mestre em Ciências da Saúde. Doutoranda em Ciências da Saúde pelo Programa de Pós-Graduação em Enfermagem da Universidade Federal de São Carlos (UFSCar). E-mail: isabela.machado1@gmail.com.

3 Graduada em Enfermagem. Doutora em Ciências. Professora Associada da Universidade Federal de São Carlos (UFSCar), vinculada ao Departamento de Gerontologia. E-mail: forlandi@ufscar.br.

4 Graduada em Serviço Social. Doutora em Serviço Social. Professora Associada da Universidade Federal de São Carlos (UFSCar), vinculada ao Departamento de Gerontologia. E-mail: marisam@ufscar.br. 
Katz e Escala de Lawton e Brody. Resultados: Participaram do estudo 28 idosos, com média de idade de 73,6 ( \pm 5,95) anos, 64,3\% pertenciam ao sexo feminino e 85,7\% possuíam renda de um salário mínimo. Houve prevalência de idosos com pré-fragilidade, 42,9\%. A fragilidade se correlacionou com a cognição (r: -0,452, p-valor: 0,018) e funcionalidade, segundo Índice de Katz (r: -0,398, p-valor: 0,036) e Escala de Lawton e Brody (r: -0,57, p-valor: 0,002). Conclusão: Os resultados sugerem que idosos jovens estão apresentando condição de pré-fragilidade. Houve correlação das atividades de vida diária e cognição com a fragilidade, achado que compromete a condição de independência para idosos morarem sozinhos em condomínio.

palavras - chave

Idoso Fragilizado. Habitação. Envelhecimento.

\section{Introdução}

Com o aumento da expectativa de vida, preocupações em relação à moradia e ao envelhecimento surgem com a necessidade de cuidados de longa duração. Os condomínios para idosos de baixa renda são opções, sendo também alternativa para os governos ao atendimento a idosos carentes ou em condição de vulnerabilidade socioeconômica (MINCACHE et al., 2010). Essa situação pode se tornar complexa, quando o processo de fragilização acompanha os idosos e os expõe a riscos e desfechos negativos relacionados à saúde, ao uso de serviços e às dependências (CESARI et al., 2014). Neste contexto, destaca-se que as novas formas de morar contemplem as necessidades de idosos frágeis e proporcionem condições adequadas de cuidados em moradia a longo prazo.

Estudos demonstram que a fragilidade é associada a situações socioeconômicas desfavoráveis, baixa escolaridade e pobreza (CESARI et al., 2014). Diante da população que vem envelhecendo, as próprias pessoas idosas preferem envelhecer na comunidade e viver de forma independente no lar pelo maior tempo possível ou até a morte. Este cenário torna-se inquietante, uma vez que pessoas com fragilidade sofrem com as necessidades decorrentes de suas condições funcionais nos aspectos físicos, psíquicos e sociais (LOOMAN et al., 2016).

Atualmente, há condomínios para idosos, implantados por meio de políticas públicas habitacionais, com objetivo de atender a idosos de baixa renda, que recebem até um salário mínimo, em situação de vulnerabilidade social, que 
vivem sozinhos ou que possuem vínculos familiares enfraquecidos e que sejam independentes na realização de suas atividades de vida diária (SÃO PAULO, 2010). A existência de políticas públicas com essas características se figura como conquista importante para questão do envelhecimento e moradia, porém estas estão sendo planejadas e desenvolvidas considerando apenas o acolhimento de idosos ativos e independentes. Logo, uma problemática pode se desenvolver pelo fato de idosos ingressarem no projeto em condições desfavoráveis para morarem sozinhos e, a curto prazo, tornarem-se frágeis e dependentes. Diante disto, torna-se relevante avaliações que identifiquem a condição de fragilidade e a necessidade de cuidados desses moradores para que possam ser assistidos integralmente pelo serviço.

Estudos acerca da fragilidade em idosos que moram na comunidade apontam que são necessárias medidas baseadas em políticas de prevenção e redução dos níveis de fragilidade (LOOMAN et al., 2016; PUTS et al., 2016). Em relação aos idosos que moram na comunidade em condomínios exclusivos, a literatura revela escassos estudos que se propõem investigar o processo de fragilidade em moradores dessa modalidade. Estudo realizado com 50 idosos de condomínio do município de Maringá, Paraná, Brasil, descreveu a utilização de serviços de saúde de residentes dessa modalidade habitacional e apontou influência positiva, como o fato de contar com unidade de atenção básica da saúde no contexto próximo ao local de residência, bem como com oferta de atividades físicas no condomínio, dados que constituem fatores protetores para manutenção da capacidade funcional e, consequentemente, para fragilidade (TESTON et al., 2013).

O modelo assistencial visa atender a idosos em situação de vulnerabilidade social e independência nas atividades diárias e que a fragilidade é presente no processo do envelhecimento e influenciada negativamente pela dificuldade no desempenho das atividades básicas e instrumentais de vida diária e pelo comprometimento cognitivo. Considerando esses fatores, este estudo objetivou identificar a relação da fragilidade com cognição, depressão e capacidade funcional de residentes em condomínio exclusivo para idosos no interior do estado de São Paulo, Brasil.

\section{Método}

Estudo transversal, com utilização do método quantitativo, realizado em condomínio exclusivo para idosos no interior do estado São Paulo, vinculado à Secretaria Municipal de Assistência e Desenvolvimento Social do município. 
As pessoas habilitadas a morar no condomínio devem atender aos critérios de inclusão: possuir 60 anos ou mais, estar em situação de vulnerabilidade socioeconômica e ser independente nas atividades de vida diária. O núcleo habitacional conta com 33 casas e um centro de convivência. No momento da pesquisa, havia residindo no local 34 idosos, quatro recusaram a participar e dois não foram encontrados na residência após quatro tentativas. A coleta de dados foi realizada nos domicílios dos idosos, por avaliador previamente treinado, e teve início após aceite e assinatura do Termo de Consentimento Livre e Esclarecido. O tempo médio de duração de cada entrevista foi de aproximadamente 90 minutos.

Foram incluídas neste estudo pessoas com 60 anos ou mais e residentes no condomínio. Os dados foram coletados de janeiro a maio de 2015, sendo as entrevistas realizadas nas residências dos idosos e no centro de convivência do condomínio.

A coleta de dados foi realizada mediante entrevista individual, em duas partes, a primeira delas continha dados sociodemográficos, como idade, sexo, renda, estado conjugal e escolaridade e a segunda, continha instrumentos para avaliação de fragilidade, cognição e atividades de vida diária. A fragilidade foi avaliada por meio do fenótipo de fragilidade, proposto por Fried e colaboradores, que avaliou cinco critérios: a) perda de peso: avaliada por meio do autorrelato do participante sobre a perda de peso não intencional nos últimos doze meses, igual ou superior a $4,5 \mathrm{~kg}$; b) fadiga: questão autorreferida avaliada por duas questões subjetivas; c) diminuição da força de preensão manual: mensurada por meio do uso do dinamômetro; d) diminuição da velocidade da marcha: avaliada pelo tempo que o idoso gasta para caminhar um percurso de 4,6 metros e; e) baixo nível de atividade física: avaliação do desempenho funcional e do nível de atividade física. O idoso foi classificado como frágil caso preenchesse três ou mais desses critérios, pré-frágil um ou dois critérios e não frágil quando não apresentasse nenhum desses critérios (FRIED et al., 2001).

A função cognitiva foi investigada por meio do Mini Exame do Estado Mental (MEEM), instrumento para rastreio de perdas cognitivas, que possui 19 itens divididos em 11 domínios: orientação temporal, orientação espacial, memória imediata, cálculo, evocação das palavras, nomeação, repetição, comando, leitura, frase e cópia do desenho. Além disso, para este estudo, utilizou-se das notas de corte relacionadas à escolaridade, sugeridas por Brucki e colaboradores (2003). A nota de corte para analfabetos foi de 17 pontos; para aqueles que possuíam de um a quatro anos de escolaridade, a nota de corte foi de 22 pontos; de um a cinco anos de escolaridade, 24 pontos; e acima de 
nove anos de escolaridade, 26 pontos. A pontuação abaixo da nota de corte foi indicativo de perda cognitiva.

A avaliação dos sintomas depressivos foi verificada por meio da Escala de Depressão Geriátrica Abreviada (GDS-15) e tem como objetivo rastrear sintomas depressivos em idosos. Elaborada por Yesavage e colaboradores, em 1983, e validada para versão brasileira por Osvaldo Almeida e Shirley Almeida, em 1999. O teste possui versões com 30 e 15 questões, para essa versão abreviada, a pontuação final foi classificada como: zero a cinco pontos, normal; seis a dez pontos, depressão leve; e onze a quinze pontos, depressão severa (BRITO; NUNES; YUASO, 2007).

O Índice de Katz foi utilizado para avaliar as atividades de vida diária. Instrumento desenvolvido por Katz e colaboradores (1963) e adaptado transculturalmente para o Brasil por Lino e colaboradores (2008) avaliou seis funções básicas: banho, vestir-se, ir ao banheiro, transferência, continência e alimentação. O Índice de Katz foi pontuado no formato Likert, em que cada tarefa recebeu pontuação específica: zero a dois pontos equivalente à dependência importante; três a quatro, dependências parciais; e cinco a seis, pontos independência.

Para avaliação das atividades instrumentais de vida diária, foi utilizada a Escala de Lawton e Brody, desenvolvida por Lawton e Brody (1969), com adaptação e validação para o Brasil por Santos e Virtuoso Jr. (2008). Esta escala possui sete itens: uso do telefone, realização de viagens e compras, preparo de refeições, trabalho doméstico, uso de medicamentos e administração do dinheiro. A pontuação varia de zero a 21 pontos, na qual 21 pontos, independência; mais que sete e menos que 21 pontos, dependência parcial; e sete pontos, dependência total.

Os dados foram analisados pelo programa estatístico Statistical Package for the Social Sciences (SPSS) versão 20.0. Foram realizadas análises descritivas como medidas de centralidade (frequência simples, absoluta e média) e medidas de dispersão (desvio padrão). Para comparações entre os grupos de fragilidade, foi utilizado o teste de Qui-Quadrado e para correlações entre a fragilidade e as variáveis contínuas, realizou-se o teste não paramétrico de Spearman. Foi considerado nível de significância $\leq 0,05$.

Os preceitos éticos foram respeitados, conforme a Resolução 466/2012. O projeto foi aprovado pelo Comitê de Ética em Pesquisa em Seres Humanos da Universidade Federal de São Carlos, de acordo com parecer 726.471/2014, e aprovado pela Secretaria Municipal de Assistência e Desenvolvimento Social do município. 
Participaram do estudo 28 idosos, com média de idade igual a 73,68 anos $( \pm 5,951)$, pertencentes ao sexo feminino $(64,3 \%)$, possuíam fundamental incompleto $(64,3 \%)$, com renda de um salário mínimo $(85,7 \%)$ e sem parceiros $(85,7 \%)$. Quarenta e dois por cento dos idosos faziam uso de cinco a seis medicamentos, $74,1 \%$ não apresentaram comprometimento cognitivo, $75 \%$ não apresentaram sintomas depressivos e $42,9 \%$ estavam pré-frágeis. Em relação aos critérios do fenótipo da fragilidade que apresentaram maiores perdas entre os participantes, destacaram-se: baixo nível de atividade física, 46,4\% $(\mathrm{n}=13)$; baixa força de preensão manual, $32,1 \%(\mathrm{n}=9)$; fadiga autorrelatada, $25 \%(n=7)$; diminuição da velocidade da marcha, $21,4 \%(n=6)$; e perda de peso não intencional, 21,4\% $(n=6)$. Quanto à funcionalidade, a maioria dos entrevistados deste estudo se mostraram independentes para atividades de vida diária. A Tabela 1 apresenta os dados de caracterização.

Tabela 1 - Distribuição das características sociodemográficas, fragilidade, cognição e funcionalidade de idosos residentes em condomínio. Araraquara, SP, 2016 ( $n=28)$.

\begin{tabular}{llll}
\hline Classificação & Variáveis & Categoria & n (\%) \\
\hline Dados sociodemográficos & Gênero & Masculino & $10(35,7)$ \\
& & Feminino & $18(64,3)$ \\
& Idade (anos) & $65-75$ & $18(64,3)$ \\
& Escolaridade (anos) & Analfabeto & $10(35,7)$ \\
& & Fundamental incompleto & $18(64,3)$ \\
& Renda individual (SM) & 1 & $5(17,9)$ \\
& & $>1$ & $24(85,7)$ \\
& Estado civil & Com parceiro & $4(14,3)$ \\
\hline \multirow{2}{*}{ Condições de saúde } & Número de & Sem parceiro & $4(14,3)$ \\
& medicamentos & $1-2$ & $24(85,7)$ \\
\hline & & $3-4$ & $3(10,7)$ \\
& & $5-6$ & $9(32,1)$ \\
& & $12(42,9)$ \\
\end{tabular}




\begin{tabular}{|c|c|c|c|}
\hline Classificação & Variáveis & Categoria & n (\%) \\
\hline & \multirow[t]{2}{*}{ Cognição } & $\begin{array}{l}\text { Com comprometimento } \\
\text { cognitivo }\end{array}$ & $7(25,9)$ \\
\hline & & $\begin{array}{l}\text { Sem comprometimento } \\
\text { cognitivo }\end{array}$ & $20(74,1)$ \\
\hline & \multirow[t]{2}{*}{ Sintomas depressivos } & Normal & $21(75)$ \\
\hline & & Depressão leve & $7(25)$ \\
\hline & \multirow[t]{3}{*}{ Fragilidade } & Não frágil & $9(32,1)$ \\
\hline & & Pré-frágil & $12(42,9)$ \\
\hline & & Frágil & $7(25)$ \\
\hline \multirow[t]{5}{*}{ Funcionalidade } & \multirow[t]{3}{*}{$\begin{array}{l}\text { Atividades básicas } \\
\text { de vida diária }\end{array}$} & Independência & $26(92,9)$ \\
\hline & & Dependência parcial & $1(3,6)$ \\
\hline & & Dependência importante & $1(3,6)$ \\
\hline & \multirow[t]{2}{*}{$\begin{array}{l}\text { Atividades instrumentais } \\
\text { de vida diária }\end{array}$} & Independência & $19(67,9)$ \\
\hline & & Dependência parcial & $9(32,1)$ \\
\hline
\end{tabular}

Nota: SM: salário mínimo

Fonte: Elaborada pelas autoras.

Quanto à comparação da fragilidade com dados sociodemográficos, cognição, sintomas depressivos e funcionalidade, obteve-se nível de pré-fragilidade na proporção de $44,4 \%$ para mulheres, $50 \%$ para os que tinham idades entre 65 e 75 anos, fundamental incompleto (35,7\%), renda de um salário mínimo (42,9\%), sem parceiros $(46,4 \%)$, com comprometimento cognitivo (37\%), sintomas depressivos normais (35,7\%) e independentes para o Índice de Katz (56\%) e Escala de Lawton e Brody $(39,3 \%)$. Realizada correlação entre a fragilidade com as outras variáveis, a fragilidade se correlacionou com uso de medicamentos (r: -0,410, p-valor: 0,042), cognição (r: -0,452, p-valor: 0,018) e funcionalidade, segundo Índice de Katz (r: -0,398, p-valor: 0,036) e Escala de Lawton e Brody (r: -0,57, p-valor: 0,002), apresentando resultados estatisticamente significantes. A depressão se correlacionou com a fragilidade, mas não apresentou significância. A Tabela 2 apresenta os achados. 


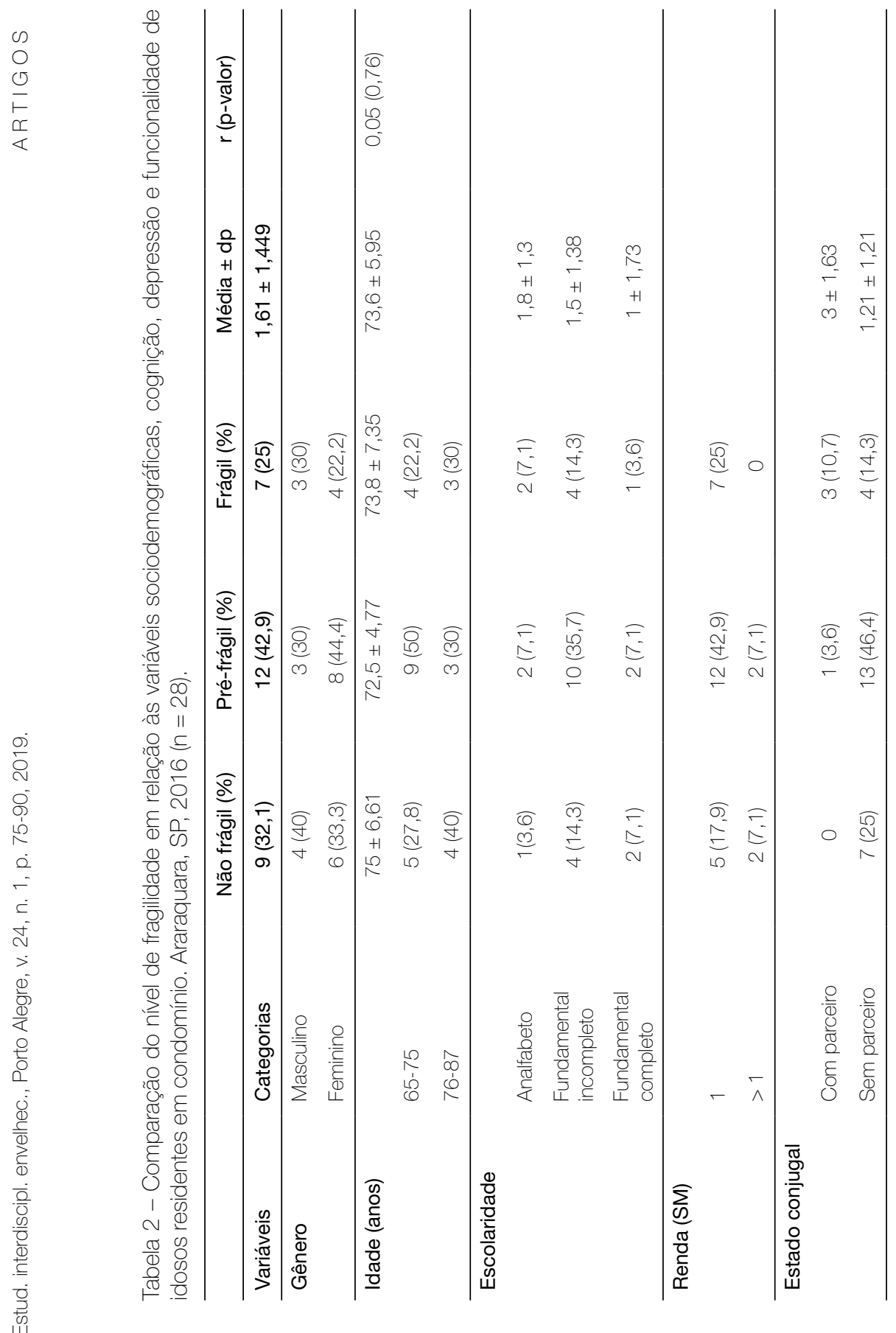




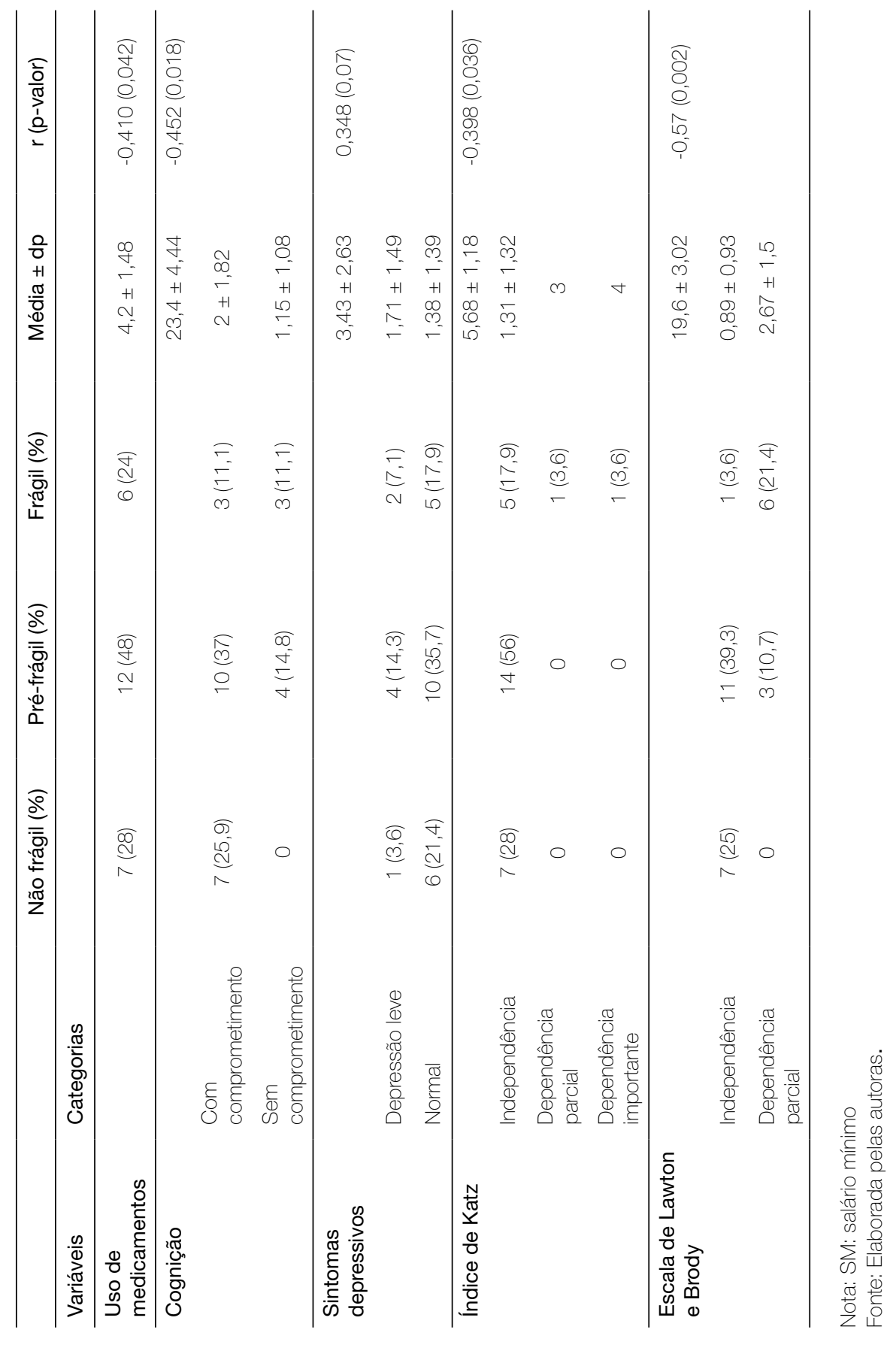

0
0
0
$\leftarrow$
$\square$
$\varangle$ 
Os idosos deste estudo apresentaram média de idade correspondente a outros estudos brasileiros que propuseram avaliar a fragilidade, com idade média equivalente a 72,7, 74,3 e 72,28 anos, porém os resultados da fragilidade dos idosos em relação à idade comportaram-se de maneira oposta à tendência encontrada na literatura, pois, no presente estudo, verificou-se que os idosos mais jovens eram mais frágeis do que os mais velhos (NERI et al., 2013; VIEIRA et al., 2013; COSTA; NERI, 2011). Da mesma forma, a variável sexo também se comportou de maneira esperada em relação ao que é encontrado na literatura. Em estudo realizado no Rio de Janeiro, com idosos de comunidade, identificou prevalência do sexo feminino, em 67,1\% (PEREZ; LOURENÇO, 2013). Outra investigação realizada com idosos no México, também, encontrou dados semelhantes, pois houve prevalência de indivíduos do sexo feminino, $64,2 \%$ (ESPINOZA; HAZUDA, 2015). A literatura apresenta que a identificação de fragilidade em pessoas com idade avançada e do sexo feminino são fatores determinantes para essa condição (CESARI et al., 2014). Outros autores também trazem contribuição, uma vez que constataram que a prevalência da fragilidade intensifica na medida em que os idosos aumentam de idade (FRIED et al., 2001).

No que diz respeito ao estado conjugal e escolaridade dos idosos deste estudo, conforme apresentado, 85,7\% não possuíam parceiro e 64,3\% não concluíram o ensino fundamental. No estudo de Neri e colaboradores (2013), o estado conjugal mais frequente foi o sem parceiro (52\%) e fundamental incompleto (64,3\%). Santos et al. (2013) também trouxeram dados semelhantes, pois $55,5 \%$ dos idosos não possuíam parceiro e $49,2 \%$ tinham de um a quatro anos de estudo. Evidências apontam que o nível de escolaridade é fator preditivo para efeitos adversos na saúde das pessoas idosas (GUTIÉRREZ-ROBLEDO; ÁVILA-FUNES, 2012). Além disso, idosos da comunidade tendem a apresentar baixa escolaridade, ocorrências de problemas de saúde mental, condições crônicas, fragilidade além de exclusão social e menor acesso às informações (SANTOS et al., 2013).

Em relação à renda, 85,7\% dos entrevistados neste estudo recebiam um salário mínimo. Observou-se, neste trabalho, que os dados divergiram dos achados da literatura. Estudo realizado com idosos da comunidade, pela Rede Perfil de Fragilidade de Idosos Frágeis (FIBRA), identificou que 54,1\% dos participantes recebiam mais de um salário mínimo, com média de renda de 4,9 salários (NERI et al., 2013; PEREZ; LOURENÇO, 2013). Uma hipótese para que esses dados se comportem dessa maneira, seria pelo fato de tratar 
de idosos que no momento do ingresso à instituição estavam em situação de vulnerabilidade social e econômica. Em maioria, esses idosos recebem como fonte de renda um salário mínimo.

A avaliação da fragilidade realizada neste estudo revelou que os idosos moradores de condomínio apresentaram, mais frequentemente, a condição de pré-fragilidade $(42,9 \%)$, seguidos pelos não frágeis $(32,1 \%)$ e frágeis $(25 \%)$. Os achados do presente estudo também corroboram com pesquisa realizada em sete localidades brasileiras, em que $51,9 \%$ dos idosos eram pré-frágeis, $39,1 \%$ não frágeis e $9 \%$ frágeis. Em estudo realizado em Belo Horizonte, MG, Brasil, também foram encontrados resultados semelhantes, pois $46,3 \%$ deles estavam pré-frágeis, $45 \%$ não estavam frágeis e $8,7 \%$ estavam fragilizados (BRUCKI et al., 2003; NERI et al., 2013; VIEIRA et al., 2013). Quanto aos dados de idosos pré-frágeis no presente estudo, enfatiza-se que se trata de idosos da comunidade que moram em mesmo local, um conjunto habitacional destinado para essa população. Andrew e colaboradores (2012) argumentam que a fragilidade pode ser influenciada por determinantes sociais, dentre estes a situação socioeconômica, pois a vulnerabilidade social pode influenciar no risco de resultados adversos de saúde, bem como na sobrevivência de idosos, incapacidades e risco precoce para óbito (ZARULLI, 2016; WALLACE et al., 2015). Desse modo, para o idoso fragilizado em situação de vulnerabilidade, há necessidade de o local de moradia assegurar-lhe proteção de vida que preze pela integridade, dignidade e autonomia.

No que diz respeito às variáveis que correlacionaram à fragilidade dos idosos, os resultados estatísticos mostraram que quanto mais frágeis, pior é o desempenho em relação à capacidade cognitiva, bem como a realização de atividades básicas e instrumentais de vida diária. A cognição, por meio do MEEM, também mostrou relação com a fragilidade, os resultados apontaram que quanto maior o grau de fragilidade do idoso, menor a pontuação no MEEM.

Os achados dos residentes desse condomínio corroboram com revisão sistemática acerca do fenótipo da fragilidade e a predição de mortalidade, em que destaca a associação entre o nível de fragilidade e desfechos intermediários, como comorbidades, dependência e declínio cognitivo (CHANG; PEI-LING, 2015). Santos e colaboradores (2013) compararam grupos de idosos frágeis, pré-frágeis e não frágeis em relação ao desempenho cognitivo e encontraram resultados estatisticamente significativos semelhantes ao presente estudo, pois os idosos mais fragilizados apresentaram menores pontuações no MEEM ( $\mathrm{p}$-valor $=0,014)$. No estudo de Leonardo e colaboradores (2014), a partir da correlação de Pearson, os pesquisadores encontraram coeficiente de correlação equivalente a - 0,513 e p-valor de 0,000 , ou seja, identificaram correlação negativa entre fragilidade e 
cognição, sendo este achado semelhante ao deste estudo, pois quanto maior o nível de fragilidade, menor a pontuação do MEEM. A literatura aponta que a presença de déficit cognitivo ocasiona dificuldades na rotina de idosos, além de alterações na autoestima e qualidade de vida (DINIZ et al., 2013). Além disso, com o envelhecimento, tende-se a ocorrer diminuição da capacidade cognitiva em pessoas idosas, podendo ser impulsionado por fatores genéticos, culturais, hábitos de vida e presença de comorbidades (ZORTEA et al., 2015).

A variável depressão não apresentou significância com a fragilidade no presente estudo. Além disso, os idosos também não apresentaram perdas nessa condição, pois apresentaram normalidade nos sintomas depressivos. Esses achados diferem dos encontrados em estudo que propôs avaliar os sintomas depressivos em moradores de condomínio de idosos de Maringá, PR, Brasil. Nessa pesquisa, identificou-se que $84 \%$ dos idosos apresentaram indícios de depressão leve e que $16 \%$ possuíam sintomas de depressão severa (TESTON; CARREIRA; MARCON, 2014). A literatura apresenta que com a redução da capacidade cognitiva, há influências no humor, associando-se com a depressão ao longo da vida (PAULA et al., 2013). Nessa perspectiva, a condição de fragilidade apresenta-se como ciclo ou espiral de declínios causada por alterações fisiopatológicas que podem ser influenciadas por doenças, demências e transtornos de humor (DUARTE; LEBRÃO, 2011).

Em relação à funcionalidade, os resultados deste estudo são semelhantes aos encontrados em outros estudos sobre fragilidade de idosos que também utilizou o Índice de Katz e a Escala de Lawton e Brody, os idosos mais fragilizados apresentaram piores condições de realização dessas atividades em relação aos idosos menos fragilizados (VIEIRA et al., 2013). Esses achados indicam que, de modo geral, na medida em que o nível de fragilidade aumenta, os idosos apresentam pior desempenho para atividades básicas e instrumentais de vida diária.

Estudo de Lustosa et al. (2013) investigou a relação da fragilidade e capacidade funcional de idosos participantes em grupo de convivência de Belo Horizonte MG, Brasil, os resultados apresentados mostraram relação entre fragilidade e atividades de vida diária (r: -0,32, p-valor de 0,001). Os achados revelaram que os idosos apresentaram condição de pré-fragilidade, pois, primeiramente, perderam a capacidade de realizar atividades de vida diária, ou seja, aquelas que requerem maiores habilidades e complexidades.

Destaca-se que idosos residentes em condomínios precisam da oferta de moradia assistida e serviços sócio assistenciais. Diante da mudança do perfil de população acolhida em condomínios municipais, estruturas organizacionais, quanto aos objetivos, critérios de inclusão e serviços oferecidos, necessitam de 
readequações para o atendimento a idosos frágeis, visando a permanência destes por mais tempo na moradia. Enfatiza-se que esses condomínios são uma das alternativas existentes em relação às modalidades de atendimento à moradia para velhice, sugere-se redirecionamento para essas modalidades, por meio da Política Habitacional, com oferecimento de outras opções de residências que atendam às necessidades e ao perfil do público-alvo.

Destaca-se como limitação do estudo o número de participantes e a impossibilidade de comparar com outros estudos que tenham avaliado fragilidade em contextos de residências exclusivas para idosos. Por tal motivo, considera-se relevante a continuidade e ampliação de outras investigações, tanto em residências quanto em serviços exclusivos para idosos.

\section{Conclusão}

Nesse estudo, houve predominância de idosos com pré-fragilidade. A fragilidade se correlacionou com a cognição e a capacidade funcional. Considera-se urgente a atenção à população idosa usuária dessa modalidade de atendimento e necessidade de readequações no acolhimento de idosos frágeis ou em processo de fragilização. Sugere-se assistência assistida, por meio dos serviços para principais necessidades desse público, bem como otimização de estratégias preventivas, dando oportunidade para idosos residirem por mais tempo nas moradias.

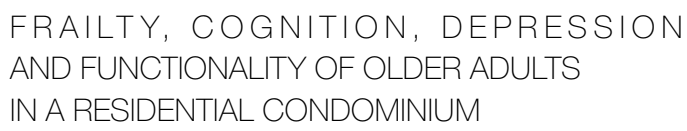

\section{abstract}

Objective: To identify the relationship of frailty with cognition, depression and functional capacity of residents in exclusive condominium for elderly people in the state of São Paulo. Method: Cross-section study using quantitative method. We used a semi-structured interview, the phenotype of frailty proposed by Fried, the Mini-Mental State Examination, Geriatric Depression Scale, Katz and the Scale of Lawton and Brody. Results: 28 elderly individuals participated in the study, with an average age of 73.6 (5.95 \pm ) years, 64.3\% were female and $85.7 \%$ had income of a minimum wage. There was prevalence of elderly pre frail, 42.9\%. Frailty correlated with cognition ( $r:-0.452, p$-value: 0.018 ) and 
functionality, according to Katz Index (r: -0.398, p-value: 0.036) and Lawton and Brody (r: -0.57, p-value: 0.002). Conclusion: The results suggest that the young elderly are showing pre frailty condition. There was correlation of activities of daily life and cognition with the frailty, which compromises the independence condition for the elderly living alone in the condominium.

keywords

Frail Elderly. Housing. Aging.

\section{referências}

ALMEIDA, Osvaldo P.; ALMEIDA, Shirley A. Reliability of the Brazilian version of the Geriatric Depression Scale (GDS) short form. Arquivos de Neuro-psiquiatria, São Paulo, v. 57, n. 2B, p. 421-426, 1999.

ANDREW, Melissa Kethryn et al. The impact of social vulnerability on the survival of the fittest older adults. Age and Ageing, London, v. 41, n. 2, p. 161-165, Mar. 2012.

BRITO, Francisco Carlos; NUNES, Maria Inês; YUASO, Denise Rodrigues. Multidimensionalidade em Gerontologia II: instrumentos de avaliação. In: NETTO, M. P. (org.). Tratado de Gerontologia. 2. ed. São Paulo: Atheneu, 2007. p. 133-147.

BRUCKI, Sonia Maria Dozzi et al. Sugestões para o uso do Mini-Exame do Estado Mental no Brasil. Arquivos de Neuro-Psiquiatria, São Paulo, v. 61, n. 3-B, p. 777-781, abr. 2003.

CESARI, Matteo et al. Sarcopenia and physical frailty: two sides of the same coin. Frontiers in Aging Neuroscience, Lausanne, v. 6, p. 192, 2014.

CHANG, Shu-Fang; PEI-LING, Lin. Frail phenotype and mortality prediction: a systematic review and meta-analysis of prospective cohort studies. International Journal of Nursing Studies, United Kingdom, v. 52, n. 8, p. 1362-1374, 2015.

COSTA, Taiguara Bertelli; NERI, Anita Liberalesso. Medidas de atividade física e fragilidade em idosos: dados do FIBRA Campinas, São Paulo, Brasil. Cadernos de Saúde Pública, Rio de Janeiro, v. 27, n. 8, p. 1537-1550, 2011.

DINIZ, Ariane Brito et al. Avaliação da cognição, atividade física e aptidão física de idosos: uma revisão crítica. Estudos de Psicologia, Natal, v. 18, n. 2, p. 315-324, 2013.

DUARTE, Yeda Aparecida Oliveira; LEBRÃO, Maria Lúcia. Fragilidade e envelhecimento. In: FREITAS, Elizabete Viana et al. (org.). Tratado de Geriatria e Gerontologia. 3. ed. Rio de Janeiro: Guanabara Koogan, 2011.

ESPINOZA, Sara; HAZUDA, Helen. Frailty prevalence and neighborhood residence in older Mexican Americans: the San Antonio longitudinal study of aging. Journal of the American Geriatrics Society, New York, v. 63, n. 1, p. 106-111, Jan. 2015.

FRIED, Linda et al. Frailty in older adults: evidence for a phenotype. Journal of Gerontology: Medical Sciences, New York, v. 56A, n. 3, p. M146-M156, 2001.

GUTIÉRREZ-ROBLEDO, Luis Miguel; ÁVILA-FUNES, Alberto. How to include the social factor for determining frailty? The Journal of Frailty \& Aging, Auzeville-Tolosane, v. 1, n. 1, p. 13-17, Feb. 2012 
KATZ, Sidney et al. Studies of illness in the aged. The Index of ADL: a standardized measure of biological and psychosocial function. The Journal of the American Medical Association, Chicago, v. 185, n. 12, p. 914-919, 1963

LAWTON, Powell; BRODY, Elaine M. Assessment of older people: self-maintaining and instrumental activities of daily living. The Gerontologist, Washington, D.C., v. 9, n. 3, p. 179-186, Autumn 1969.

LEONARDO, Kizie Conrado et al. Avaliação do estado cognitivo e fragilidade em idosos mais velhos, residentes no domicílio. Ciência, Cuidado e Saúde, Maringá, v. 13, n. 1, p. 120-127, 2014.

LINO, Valéria Teresa Saraiva et al. Adaptação transcultural da Escala de Independência em Atividades da Vida Diária (Escala de KATZ). Cadernos de Saúde Pública, Rio de Janeiro, v. 24, n. 1, p. 103-112, 2008.

LOOMAN, Wilhelmina Mijntje et al. The effects of a pro-active integrated care intervention for frail community-dwelling older people: a quasi-experimental study with the GP-practice as single entry point. BMC Geriatrics, London, v. 16, Feb. 2016.

LUSTOSA, Lygia Paccini et al. Fragilidade e funcionalidade entre idosos frequentadores de grupos de convivência em Belo Horizonte, MG. Revista Brasileira de Geriatria e Gerontologia, Rio de Janeiro, v. 16, n. 2, p. 347-354, jun. 2013.

MINCACHE, Gisnelli Bataglia et al. Sensações do morar e a concretização de moradia para idosos egressos em um albergue. Caderno Temático Kairós Gerontologia, São Paulo, v. 8, p. 169-193, 2010.

NERI, Anita Liberalesso et al. Metodologia e perfil sociodemográfico, cognitivo e de fragilidade de idosos comunitários de sete cidades brasileiras: Estudo FIBRA. Cadernos de Saúde Pública, Rio de Janeiro, v. 29, n. 4, p. 778-792, jan. 2013.

PAULA, Ana Flávia Marostegan de et al. Avaliação da capacidade funcional, cognição e sintomatologia depressiva em idosos atendidos em ambulatório de Geriatria. Revista da Sociedade Brasileira de Clínica Médica, São Paulo, v. 11, n. 3, p. 212-218, 2013.

PEREZ, Mariangela; LOURENÇO, Roberto Alves. Rede FIBRA-RJ: fragilidade e risco de hospitalização em idosos da cidade do Rio de Janeiro, Brasil. Cadernos de Saúde Pública, Rio de Janeiro, v. 29, n. 7, p. 1381-1391, mar. 2013.

PUTS, Martine et al. Interventions to prevent or reduce the level of frailty in community-dwelling older adults: a protocol for a scoping review of the literature and international policies. BMJ Open, London, v. 6, n. 3, e010959, 2016.

SANTOS, Ariene Angelini dos et al. Sono, fragilidade e cognição: estudo multicêntrico com idosos brasileiros. Revista Brasileira de Enfermagem, Brasília, DF, v. 66, n. 3, p. 351-357, maio/jun. 2013.

SANTOS, Roberto Lopes dos; VIRTUOSO JÚNIOR, Jair Sindra. Confiabilidade da versão brasileira da Escala de Atividades Instrumentais da Vida Diária. Revista Brasileira em Promoção da Saúde, v. 21, n. 4, p. 290-296, 2008.

SÃO PAULO (Estado). Decreto n 56.448, de 29 de novembro de 2010. Dá nova redação a dispositivos do Decreto n 54.285, de 29 de abril de 2009 que autoriza as Secretarias Estaduais da Habitação e de Assistência e Desenvolvimento Social, representando o Estado, a celebrar convênios com a Companhia de Desenvolvimento Habitacional e Urbano do Estado de São Paulo - CDHU e com os Municípios do Estado de São Paulo, visando a implementação do Programa Vila Dignidade. São Paulo: Assembleia Legislativa do Estado de São Paulo, 2010. Disponível em: http://www.al.sp.gov.br/repositorio/ legislacao/decreto/2010/decreto-56448-29.11.2010.html. Acesso em: 25 mar. 2018.

TESTON, Elen Ferraz; CARREIRA, Ligia; MARCON, Sonia Silva. Sintomas depressivos em idosos: comparação entre residentes em condomínio específico para idoso e na comunidade. Revista Brasileira de Enfermagem, Brasília, DF, v. 67, n. 3, p. 450-456, maio/jun. 2014. 
TESTON, Elen Ferraz et al. Use of health services by residents at a seniors-only living facility. Revista da Escola de Enfermagem da USP, São Paulo, v. 47, n. 5, p. 1122-1128, out. 2013.

VIEIRA, Renata Alvarenga et al. Prevalência de fragilidade e fatores associados em idosos comunitários de Belo Horizonte, Minas Gerais, Brasil: dados do Estudo FIBRA. Cadernos de Saúde Pública, Rio de Janeiro, v. 29, n. 8, p. 1631-1643, ago. 2013.

WALLACE, Lindsay et al. Social vulnerability as a predictor of mortality and disability: cross-country differences in the survey of health, aging, and retirement in Europe (SHARE). Aging: Clinical and Experimental Research, v. 27, n. 3, p. 365-372, 2015.

ZARULLI, Virginia. Unobserved heterogeneity of frailty in the analysis of socioeconomic differences in health and mortality. European Journal of Population, Netherlands, v. 32 , n. 1, p. 55-72, Jan. 2016.

ZORTEA, Bruna et al. Avaliação cognitiva de pessoas idosas em atendimento ambulatorial. Revista Rene, Fortaleza, v. 16, n. 1, p. 123-131, jan./fev. 2015. 